\title{
Grain boundary energies in body-centered cubic metals
}

Sutatch Ratanaphan ${ }^{1,2}$, David L. Olmsted ${ }^{3}$, Vasily V. Bulatov ${ }^{4}$, Elizabeth A. Holm ${ }^{1}$, Anthony D. Rollett ${ }^{1}$, and Gregory S. Rohrer ${ }^{1}$

${ }^{1}$ Department of Materials Science and Engineering, Carnegie Mellon University, Pittsburgh, PA 15213-3890 U.S.A.

${ }^{2}$ (Current address:) Department of Tool and Materials Engineering, King Mongkut's University of Technology Thonburi, 126 Pracha Uthit Rd, Thung Khru, Bangkok 10140, Thailand

${ }^{3}$ Department of Materials Science and Engineering, University of California, Berkeley, CA 94720 U.S.A.

${ }^{4}$ Lawrence Livermore National Laboratory, 7000 East Avenue, Livermore, CA 94550, USA.

\begin{abstract}
Atomistic simulations using the embedded atom method were employed to compute the energies of 408 distinct grain boundaries in BCC Fe and Mo. This set includes grain boundaries that have tilt, twist, and mixed character and coincidence site lattices ranging from $\Sigma 3$ to $\Sigma 323$. The results show that grain boundary energies in Fe and Mo are influenced more by the grain boundary plane orientation than by the lattice misorientation or lattice coincidence. Furthermore, grain boundaries with (110) planes on both sides of the boundary have low energies, regardless of the misorientation angle or geometric character. Grain boundaries of the same type in Fe and Mo have strongly correlated energies that scale with the ratio of the cohesive energies of the two metals.
\end{abstract}




\section{Introduction}

Because grain boundaries have a significant influence on the physical properties of polycrystalline solids [1-4], their properties, including energy, have been the subjects of extensive experimental and computational study. In annealed polycrystals, the grain boundary energy distribution (GBED) is known to be inversely correlated to the grain boundary character distribution (GBCD), defined as the relative areas of grain boundaries distinguished by lattice misorientation and grain boundary plane orientation $[5,6]$. Morawiec [7] developed a technique to determine the GBED from three-dimensional electron backscatter diffraction (3D-EBSD) data, and this has been applied to measure grain boundary energies in a number of ceramics and metals including $\mathrm{MgO}[8,9], \mathrm{Y}_{2} \mathrm{O}_{3}$ [10], Ni [11], a Ni-based alloy [12], a ferritic steel [13], and an austenitic steel [14]. This method requires large amounts of data because there are no assumptions about the functional form of the GBED, and the number of unknown energies scales with the discretization of the system. Furthermore, the results are relative, rather than absolute, values of the grain boundary energies. Nevertheless, to the extent that comparisons of the measured and calculated energies have been possible, there has been satisfactory agreement between experiment and simulation, especially for the most frequently observed grain boundaries in the materials examined ( $\Sigma 3$ and $\Sigma 9$ boundaries) $[15,16]$.

The agreement between grain boundary energies derived from experiment and simulation suggests that both methods are reliable when properly applied [16]. Therefore, computer simulations by the embedded atom method (EAM) can be utilized to survey and compare a large number of grain boundary energies [17, 18]. For example, Holm et al. [17] recently showed that the grain boundary energies in $\mathrm{Al}, \mathrm{Au}, \mathrm{Cu}$, and $\mathrm{Ni}$, which share the 
face-centered cubic (FCC) crystal structure, are correlated and scale with the shear modulus. One purpose of the present study is to determine whether or not body-centered cubic (BCC) metals exhibit the same phenomenon; we therefore employ similar methods.

There have been a number of atomistic simulations of grain boundary energies in BCC metals [19-25]. Wolf [22-24] showed that the energy anisotropies of Fe and Mo were similar for symmetrical tilt boundaries, twist boundaries on (100) and (110) planes, and certain general grain boundaries. Morita and Nakashima [20] investigated the boundary energy of $<001>$ symmetric tilt boundaries in Mo, producing results consistent with the boundary energies calculated by Wolf [22-24] and with experimental boundary energies measured by the thermal grooving method [18]. Tschopp et al. [21] examined a large data set of grain boundary energies in Fe using molecular statics. Kim et al. [19] used molecular statics calculations to populate a grain boundary energy database that uniformly samples the five parameter grain boundary space for BCC Fe. While these simulations uniformly covered misorientation space in $10^{\circ}$ increments, the discretization of the grain boundary plane space was coarser.

Recently, Yesilleten and Arias [25] showed that the boundary energies of $<110>$ symmetric tilt boundaries in Mo were influenced by the incorporation of vacancies. Specifically, the coherent twin boundary energy increased from $0.61 \mathrm{~J} / \mathrm{m}^{2}$ to $2.13 \mathrm{~J} / \mathrm{m}^{2}$ when half of the atoms in the plane adjacent to the grain boundary were removed. This result illustrates the significant influence that the atomic structure of the interface can have on the computed energy. In general, if only one starting configuration is considered, there is no guarantee that the minimized energy of that boundary corresponds to the global minimum boundary energy. In their study of grain boundaries in FCC metals, Olmsted et al. 
[18] addressed this difficulty by minimizing the energies of hundreds or more of crystallographically identical boundaries that had different microscopic starting configurations. This exploration of microscopic configurations provides a greater likelihood that an energy near the minimum is reached. In general, one would expect that the naturally occurring boundaries have sufficient time and ample supply of point defects to reach a minimum of their free energies in the grand canonical ensemble.

The database created by Kim et al. [19] contains far more grain boundary energies for a BCC material than any of the other calculations. However, they considered only one initial configuration for each grain boundary type, so it is not obvious that these energies represent the minimum energy states. The purpose of this paper is to report the results of the calculation of 408 grain boundary energies in Fe and Mo. For this survey, between 100 and 10,000 different initial microscopic states were considered for each type of boundary and we report the minimum energy. We chose Fe and Mo because these materials are widely used in the polycrystalline form and there are previous reports to which we can compare subsets of the results. We also examine the correlation between the grain boundary energies in Fe and Mo and consider the extent to which isomorphic materials have correlated grain boundary energies.

\section{Methods}

This work considers the grain boundaries in all bicrystals that can be constructed in a periodic cell with dimensions less than or equal to $20 \mathrm{a}_{0} / 2$, measured parallel to the grain boundary, where $\mathrm{a}_{0}$ is the lattice spacing. The 408 boundaries that fit within this cell have 80 different misorientations and a range of characters; 176 are twist boundaries, 381 are 
tilt boundaries, and 20 are neither pure tilt nor pure twist boundaries. Note that 169 of the boundaries are both twist and tilt, depending on the choice of axis [26-28]. The 408 boundaries are not evenly distributed throughout the space of possible grain boundaries and do not provide complete coverage. For example, there are $40 \Sigma 3,27 \Sigma 5,21 \Sigma 7$, and 29 $\Sigma 9$ grain boundaries, meaning that $29 \%$ of the 408 grain boundaries are concentrated at four misorientations. Because these grain boundaries were selected based only on a maximum periodicity condition, they are of relatively high symmetry and are not expected to mimic the actual distribution of grain boundary types in real polycrystals.

Grain boundary energies were computed by minimizing system energy using the conjugate-gradient method in the LAMMPS code [29] at $\mathrm{T}=0 \mathrm{~K}$ with embedded-atom method (EAM) interatomic potentials. The computational cell has periodic boundary conditions in the $y$ and $\mathrm{z}$ directions with a minimum length of $17 \mathrm{a}_{0} / 2$. The minimum length in the $x$ direction, which is normal to the plane of grain boundary, was $20 a_{0} / 2$. The computational scheme is similar to prior studies of the grain boundary energy for FCC metals, which has been described in detail elsewhere $[17,18]$. The Mendelev potential 2 was used for $\mathrm{Fe}$ [30] and the Finnis-Sinclair potential was used for Mo [31]. These potentials were selected because they reproduced the lattice constants and the elastic constants of Fe and Mo (see Table 1), and have also been used to simulate grain boundary energies [21-24] For each macroscopic grain boundary structure, 100 to 10000 initial configurations were minimized. These initial configurations were generated using a method similar to that described by Olmsted et al. [18] with a modification to the step where atoms are removed if they were too close together. Before removing atoms, each atom in the grain boundary region was perturbed by a very small distance in a random 
direction. This displacement should have little or no effect in the current work, where atoms were removed in groups, as in ref. [18]. In the case where atoms are removed one at a time, the random displacement improves the search by preventing multiple atom pairs from being exactly at the same distance $[32,33]$. The grain boundary energy is taken to be the smallest value among the results obtained from minimizing each of a large number of initial configurations separately.

It is important to recognize that the grain boundary energy calculated here (and in all molecular statics studies) is the zero-temperature free energy, i.e. enthalpy. At finite temperatures, entropic effects cause the boundary free energy to decrease as temperature increases. While there is no experimental data for the change in free energy with temperature, atomic simulations show that the free energy decreases by about two-thirds from $0 \mathrm{~K}$ to the melting temperature [34-37]. Because this behavior seems consistent among boundaries studied [37], it is assumed that finite-temperature free energy scales with enthalpy; thus enthalpy is an appropriate measure of the relative (but not absolute) boundary free energy. However, it is likely that there are boundaries that do not follow this scaling, particularly if they undergo a structural transformation upon heating [38].

\section{Results}

The full boundary energy data set for Fe and Mo is available as online supplemental material. A comparison between the previously calculated grain boundary energies [19$25]$ and the energies of the same boundaries calculated as part of this work is shown in Fig.

1. For Fe (Fig. 1a), there is excellent agreement between our calculations and previous studies, with some scatter for the higher energy boundaries. Because Wolf [22-24] and 
Kim et al. [19] used different potentials from those used here, some differences are expected. Four of the boundary energies calculated by Wolf [22-24] and one calculated by Kim et al. [19] show a positive deviation from the present work, while those of Tschopp et al. [21] are nearly identical to our calculations. It should be noted that the present work and the previous calculation by Tschopp et al. [21] included multiple initial configurations for the same macroscopic grain boundaries. Because Wolf [22-24] and Kim et al. [19] considered only a single initial configuration for each grain boundary, their boundary energies might not represent the minimum energy structures. However, note that two of the energies reported by Wolf [22-24] and one reported by Kim et al. [19] are slightly below those calculated here. While we do not know the origin of the deviation, it is of small magnitude and occurs only for three of the highest energy boundaries. Finally, it should be noted that while Kim et al. [19] calculated the energies of many boundaries, the grain boundary plane inclinations generally deviated from the exact orientations of the boundaries considered here, so this also contributes to differences in the energies. The three included here are those cited by Kim et al. [19] as the minimum energies at $\Sigma 3$ and $\Sigma 9$ and the maximum energy at $\Sigma 3$, which we compare to the $\Sigma 3$ (112) symmetric tilt, the $\Sigma 9$ (110) twist, and the $\Sigma 3$ (111) twist, respectively.

The trend for Mo (Fig. 1b) is similar to the results for Fe. Note that when the previous calculations deviate from the present ones, they are higher in energy in all but two cases. The boundaries with small negative deviations are high energy (111) twist boundaries calculated by Wolf [22-24]. The Wolf and Morita boundaries with positive energy deviations likely result from initial atomic structures that are not close enough to the minimum energy configuration. In contrast, Yesilleten and Arias [25] did an extensive 
survey of microscopic parameters with approximately 400,000 initial configurations, so their boundary structures are presumed to be correct. The difference, in this case, is that they used an interatomic potential developed by Moriarty [43,44], rather than the FinnisSinclair potential [31] used in the present study, the previous work by Wolf [22-24], and by Morita and Nakashima [20]. The Moriarty potential appears to give higher overall boundary energies while maintaining similar relative energies between boundaries. In fact, a plot of boundary energy scaled by the coherent twin energy would show excellent agreement between our data and that of Yesilleten and Arias [25]. The consistency between our grain boundary energies and earlier calculations verifies our approach.

A small number of grain boundary energies in Fe and Mo have also been computed using density functional theory (DFT) [45-51]. These energies are uniformly larger than those computed in the present study using the embedded atom method. For example, for the $\Sigma 3$ boundary terminated on (111) planes in Fe, the grain boundary energies computed by DFT are 1.61 [45], 1.57 [51], and 1.52 [48] J/ $\mathrm{m}^{2}$ while the energy of the same boundary in the present study is $1.27 \mathrm{~J} / \mathrm{m}^{2}$. Similarly, for the $\Sigma 3$ boundary terminated on (112) planes, the grain boundary energies computed by DFT are 0.47 [47], and 0.34 [48] J/m² while the energy of the same boundary in the present study is $0.262 \mathrm{~J} / \mathrm{m}^{2}$. We presume that differences arise from the way that the two methods handle magnetism, although differences in the assumed atomic structure may also contribute. For Mo, two values have been computed by DFT for the $\Sigma 5$ (310) boundary, 1.70 [49] and $1.55[50] \mathrm{J} / \mathrm{m}^{2}$. Both of these values are somewhat larger than the value computed for the same boundary by EAM in this work, $1.33 \mathrm{~J} / \mathrm{m}^{2}$.

Figure 2 shows the calculated boundary energies of Fe and Mo as a function of 
disorientation angle (defined as the minimum misorientation angle for all rotation axes). There are no distinct trends as a function of disorientation angle, which is consistent with results in FCC metals [18]. Two specific types of boundaries are indicated by different symbols: those terminated by $\{110\}$ planes (squares) and those terminated by $\{112\}$ planes (triangles). Note that with the exception of those boundaries with a $60^{\circ}$ disorientation, each set of boundaries has a roughly constant energy, independent of disorientation angle. It is also notable that boundaries with (110) planes on either side of the interface are unusually low in energy, regardless of the disorientation angle. This trend has been observed previously [24] and supports a model of the grain boundary as the total energy of adjoining surfaces minus the binding energy $[5,6]$. Because the (110) surface has the lowest energy [52], grain boundaries composed of two (110) planes should have relatively lower grain boundary energies. However, the limitations of this simple explanation are revealed by the cases of boundaries terminated by planes with high surface energies that also have low grain boundary energies.

The grain boundary energies of Fe and Mo are plotted in Fig. 3 as a function of the $\Sigma$ value, defined as the inverse of the fraction of coincident lattice sites for a specific misorientation. The results illustrate that the $\Sigma$ value is a poor indicator of grain boundary energy, as previously noted for FCC boundaries [18]. For example, boundaries with the $\Sigma 3$ misorientation have energies that span the spectrum from the lowest to nearly the highest energy, from $0.26 \mathrm{~J} / \mathrm{m}^{2}$ and $1.27 \mathrm{~J} / \mathrm{m}^{2}$ in Fe and from $0.39 \mathrm{~J} / \mathrm{m}^{2}$ and $1.84 \mathrm{~J} / \mathrm{m}^{2}$ in Mo. Similarly, a high $\Sigma$ grain boundary can also show a large range of energies. In $\mathrm{Fe}$, the $\Sigma 43$ grain boundary has energies ranging from $0.58 \mathrm{~J} / \mathrm{m}^{2}$ to $1.32 \mathrm{~J} / \mathrm{m}^{2}$ and in Mo the energies range from $0.88 \mathrm{~J} / \mathrm{m}^{2}$ to $1.83 \mathrm{~J} / \mathrm{m}^{2}$. Even if one considers the average of the energies over 
all of the grain boundary plane orientations that were sampled (see Table 2), there is no trend to suggest that the energies scale with the lattice coincidence. Finally, we note that the mean of all of the grain boundary energies for Fe is $1.11 \pm 0.209 \mathrm{~J} / \mathrm{m}^{2}$, which compares favorably with the mean $\Sigma 3$ energy of $1.06 \pm 0.247 \mathrm{~J} / \mathrm{m}^{2}$. We also note that the mean boundary energy is on that same order as the average boundary energy of $0.985 \mathrm{~J} / \mathrm{m}^{2}$ measured in Fe at $1480{ }^{\circ} \mathrm{C}$ by Roth [53]; however, as noted above, the agreement between calculated enthalpy and measured free energy is likely fortuitous.

Finally, the variation of the boundary energy with the grain boundary plane orientation can be considered. This is only meaningful in the cases where the energies of a sufficient number of grain boundary plane orientations were calculated at constant misorientation $(\Sigma 3, \Sigma 9$, and $\Sigma 5)$. This is identical to the presentation of the grain boundary energies in the experimental study of ferrite [13] and similar to the presentation of the calculations by Kim et al. [19]. As illustrated in Fig. 4, the most significant trends for the $\Sigma 3$ misorientation are that the maximum energy occurs for the (111) twist boundary and the minima occur in the zone of orientations at $90^{\circ}$ to this position. For Fe, this agrees with the results calculated by Kim et al. [19] and the experimental study [13, 54]. The most significant trends for the $\Sigma 9$ misorientation are that the minimum energy occurs for the (110) twist boundary and the maxima occur in the zone of orientations at $90^{\circ}$ to this position. For Fe, this agrees with the results calculated by Kim et al. [19] and the results of the experimental study for the grain boundary distribution, which shows a maximum population at the minimum energy, as expected $[5,13]$. The distribution of grain boundary energies for the $\Sigma 5$ misorientation is more isotropic and biased toward higher energies in both Fe and Mo. 


\section{Discussion}

Consistent with the results of the calculated energies for FCC metals [18], the grain boundary energies of BCC metals are more sensitive to the grain boundary plane orientation than to the lattice misorientation. Subsets of the data support the hypothesis that low index planes, which have low surface energies, are often found in low energy grain boundaries. For example, (110) is the lowest energy surface, and boundaries with (110) planes on both sides have consistently low grain boundary energies $[52,55]$. There are significant counter examples, however. For the $\Sigma 3$ misorientation, the energies of the $60^{\circ}$ (110) symmetric tilt grain boundaries are slightly higher than the energies of the (112) symmetrical tilt grain boundaries (the coherent twin boundary) in both Fe and Mo. The relatively low energy of the (112) coherent twin boundary is consistent with its measured relative energy in Fe $[13,54,56]$ and Mo [57]. It should also be noted that the ratio of the coherent twin boundary energy to the average $\Sigma 3$ boundary energy in Fe $(0.25)$ and Mo (0.26) are very close to the measured energy ratio of the coherent twin boundary and ordinary grain boundaries in silicon ferrite reported as 0.22 by Dunn et al. [54].

While low energy grain boundaries often have low index planes, grain boundaries that have high index planes and low energy also appear in these data. For example, the quasi-symmetric boundary with (741) planes at the disorientation angle of $50.48^{\circ}$ has an energy $\left(0.57 \mathrm{~J} / \mathrm{m}^{2}\right.$ and $0.80 \mathrm{~J} / \mathrm{m}^{2}$ in Fe and Mo, respectively) that is even lower than the (110) twist boundary at this disorientation angle. Furthermore, there are three asymmetric $\Sigma 3$ mixed boundaries with boundary planes of $(8,4,1)(2,2,1),(11,7,1)(3,3,1)$, and $(13,11,5)(5,3,1)$ that have relatively low energies in both Fe and Mo; these boundaries 
are called out in Figs. 2 and 3. Therefore, while low energy boundaries are frequently associated with low index, low energy planes, there are exceptions, as noted above. The reasons for these exceptions likely derive from the details of boundary structure, which are beyond the scope of the present study.

Consistent with the hypothesis that grain boundary population is inversely related to energy, the $\Sigma 3$ coherent twin is the highest population boundary in both FCC and BCC polycrystals. However, where the coherent twin typically accounts for $10-50 \%$ of the grain boundary area in an annealed FCC polycrystal (c.f. [58]), the coherent twin population in ferritic steels is $3 \%$ or less (c.f. $[13,59]$ ). Our data suggest two factors that may contribute to this difference. First, the coherent twin energy is simply not as small, either in absolute or relative terms, in BCC metals as it is in FCC metals. For example, the coherent twin energy in FCC Ni is reported as $0.064 \mathrm{~J} / \mathrm{m}^{2}$, or 0.058 of the average boundary energy [18]; for BCC Fe the coherent twin has energy of $0.26 \mathrm{~J} / \mathrm{m}^{2}$, or 0.23 of the average energy. In addition, in the FCC boundary survey, the coherent twin was relatively unique in its low energy [18], whereas there are several boundaries of comparably low energy in the BCC survey, as seen in Fig. 2. Both its higher energy and the availability of other low energy boundaries could serve to decrease the relative population of coherent twins in BCC metals.

Comparing the energies in Figs. 2, 3, and 4, it is clear that the energy distributions in Fe and Mo are similar. The strong correlation between the energies of the two metals is demonstrated in Fig. 5, where each point represents one of the 408 grain boundaries and the coordinates of the point are determined by the energies of that boundary in Fe and Mo. The fact that the points fall close to a single line (with correlation coefficient 0.99) shows the strong correlation among the energies. In other words, if a certain type of grain 
boundary has a relatively low (high) energy in Fe, it will also have a relatively low (high) energy in Mo. While the coherent twin boundary has the lowest energy in both Fe and Mo, the highest boundary energies in Fe $\left(1.40 \mathrm{~J} / \mathrm{m}^{2}\right)$ and $\mathrm{Mo}\left(1.97 \mathrm{~J} / \mathrm{m}^{2}\right)$ are not associated with the same grain boundary; however, in this energy regime there are many boundaries with nearly degenerate energies. The strong correlation between the grain boundary energies of metals with the same crystal structure is consistent with what has been observed for the calculated grain boundary energies in FCC metals [17].

The scatter in the energies from the perfect correlation might derive from several sources. First, different functional forms of the EAM potentials for Fe and Mo may favor slightly different structures and therefore energies in some boundaries. Second, for each macroscopic grain boundary, the lowest energy microscopic configuration chosen from a large number of initial states might not be the same in the two metals. Finally, Fe and Mo are physically distinct, so some structural differences are expected. Considering these factors, the scatter about the line is not too surprising.

Several lines have been placed on Fig. 5 to compare the scaling of the grain boundary energies to physical parameters, including ratios of the elastic moduli, the twin boundary energy, and the cohesive energy per atom. In each case, multiples of the lattice constant have been applied to make the dimensions comparable (energy per area). The ratio of the cohesive energies $\left(\mathrm{E}_{\mathrm{coh}} / \mathrm{a}_{0}{ }^{2}\right)$, which is 1.4 , is closest to the actual scaling factor. Because a grain boundary disrupts bonds between atoms, it makes sense that the grain boundary energy would scale with the cohesive energy per atom. Indeed, this is the basis for the broken bond model of grain boundary energy [60-62]. However, this is not the same scaling seen in FCC metals, where boundary energies are observed to scale with the 
largest shear modulus $\left(\mathrm{a}_{0} \mathrm{C}_{44}\right)$ and the Voigt average shear modulus (ao $\left.\mu_{\text {voigt }}\right)$ [17]. Without results for more materials, it is not possible to gauge whether these different scaling results can be expected to apply to all FCC and BCC metals. The environment of atoms in grain boundaries spans a spectrum from perfectly crystalline to substantially distorted. The elastic moduli describe very small displacements from the perfect crystal (bond stretching), while the cohesive energy captures the complete separation between atoms (bond breaking). Therefore, grain boundary energies should be expected to have contributions from both effects. It is likely that neither the shear modulus nor the cohesive energy provides a complete model for grain boundary energy scaling.

Finally, we note that the nearly perfect scaling between the grain boundary energies in Mo and Fe is yet another strong indication that the energy anisotropy is mostly a function of the grain boundary geometry, namely of the five macroscopic degrees of freedom. Even though there may be variations in the atomistic grain boundary structure and relaxations from material to material, these differences are dwarfed by the influence of grain boundary geometry. This suggests that there is an as yet undetermined function that can be used to compute the grain boundary energy as a function of the geometric parameters, as was found for the FCC metals [54].

\section{Conclusions}

The energies of 408 grain boundaries in Fe and Mo have been calculated using embedded atom method simulations. When the initial boundary structure is properly optimized, our results agree well with previous studies. The calculated energies vary significantly with the grain boundary plane orientation, and low energy grain boundaries 
are likely to be comprised of low index planes, although low energy boundaries with high index boundary planes are also observed. The energies do not, however, show any distinct trends with misorientation angle or with the density of coincident lattice sites. The $\Sigma 3$ coherent twin has the smallest boundary energy among the boundaries surveyed; however, it is neither as small or as uniquely small as in FCC metals. The energies of grain boundaries in Fe and Mo, which share the same crystallography, are (linearly) proportional to each other with a slope proportional to the ratio of their cohesive energies, indicating that boundary structure governs energy in BCC metals.

\section{Acknowledgments}

G.S.R. and A.D.R. acknowledge support from the Office of Naval Research under MURI grant N00014-11-1-0678. D.L.O. was supported by the US Department of Energy through Grant No. DE-AC02-05CH11231. V.V.B. was supported by the US DOE Office of Basic Energy Sciences, Division of Materials Sciences and Engineering. E.A.H. was supported by the National Science Foundation under Award Number DMR-1307138. S.R. Acknowledges the Computational Chemistry \& Materials Science Summer Institute at Lawrence Livermore National Laboratory (LLNL), the Higher Educational Strategic Scholarship for Frontier Research Network, the Higher Education Research Promotion and the National Research University Project of Thailand, Office of the Higher Education Commission.

\section{References}

[1] D. Brandon. Defining grain boundaries: an historical perspective, Mater. Sci. Tech. 26 (2010) 762-773.

[2] M.P. Harmer. Interfacial Kinetic Engineering: How Far Have We Come Since Kingery's Inaugural Sosman Address?, J. Amer. Ceram. Soc. 93 (2010) 301-317. 
[3] A. Sutton, R. Balluffi. Interfaces in Crystalline Materials, Oxford University Press, Oxford, 1995.

[4] A.P. Sutton, R.W. Balluffi. Overview 61. On Geometric Criteria for Low Interfacial Energy, Acta Metal. 35 (1987) 2177-2201.

[5] G.S. Rohrer. Grain boundary energy anisotropy: a review, J. Mater. Sci. 46 (2011) 5881-5895.

[6] G.S. Rohrer. Measuring and Interpreting the Structure of Grain-Boundary Networks, J. Amer. Ceram. Soc. 94 (2011) 633-646.

[7] A. Morawiec. Method to calculate the grain boundary energy distribution over the space of macroscopic boundary parameters from the geometry of triple junctions, Acta Mater. 48 (2000) 3525-3532.

[8] D.M. Saylor, A. Morawiec, G.S. Rohrer. Distribution and energies of grain boundaries in magnesia as a function of five degrees of freedom, J. Amer. Ceram. Soc. 85 (2002) 30813083.

[9] D.M. Saylor, A. Morawiec, G.S. Rohrer. The relative free energies of grain boundaries in magnesia as a function of five macroscopic parameters, Acta Mater. 51 (2003) 36753686.

[10] S.J. Dillon, G.S. Rohrer. Characterization of the Grain-Boundary Character and Energy Distributions of Yttria Using Automated Serial Sectioning and EBSD in the FIB, J. Amer. Ceram. Soc. 92 (2009) 1580-1585.

[11] J. Li, S.J. Dillon, G.S. Rohrer. Relative grain boundary area and energy distributions in nickel, Acta Mater. 57 (2009) 4304-4311.

[12] G.S. Rohrer, J. Li, S. Lee, A.D. Rollett, M. Groeber, M.D. Uchic. Deriving grain boundary character distributions and relative grain boundary energies from three-dimensional EBSD data, Mater. Sci. Tech. 26 (2010) 661-669.

[13] H. Beladi, G.S. Rohrer. The relative grain boundary area and energy distributions in a ferritic steel determined from three-dimensional electron backscatter diffraction maps, Acta Mater. 61 (2013) 1404-1412.

[14] H. Beladi, N.T. Nuhfer, G.S. Rohrer. The five-parameter grain boundary character and energy distributions of a fully austenitic high-manganese steel using three dimensional data, Acta Mater. 70 (2014).

[15] E.A. Holm, G.S. Rohrer, S.M. Foiles, A.D. Rollett, H.M. Miller, D.L. Olmsted. Validating computed grain boundary energies in fcc metals using the grain boundary character distribution, Acta Mater. 59 (2011) 5250-5256.

[16] G.S. Rohrer, E.A. Holm, A.D. Rollett, S.M. Foiles, J. Li, D.L. Olmsted. Comparing calculated and measured grain boundary energies in nickel, Acta Mater. 58 (2010) 50635069.

[17] E.A. Holm, D.L. Olmsted, S.M. Foiles. Comparing grain boundary energies in facecentered cubic metals: Al, Au, Cu and Ni, Scripta Mater. 63 (2010) 905-908.

[18] D.L. Olmsted, S.M. Foiles, E.A. Holm. Survey of computed grain boundary properties in face-centered cubic metals: I. Grain boundary energy, Acta Mater. 57 (2009) 3694-3703.

[19] H.K. Kim, W.S. Ko, H.J. Lee, S.G. Kim, B.J. Lee. An identification scheme of grain boundaries and construction of a grain boundary energy database, Scripta Mater. 64 (2011) 1152-1155.

[20] K. Morita, H. Nakashima. Atomic periodicity of $<001>$ symmetric tilt boundary in molybdenum, Mater. Sci. Eng. A 234 (1997) 1053-1056. 
[21] M.A. Tschopp, K.N. Solanki, F. Gao, X. Sun, M.A. Khaleel, M.F. Horstemeyer. Probing grain boundary sink strength at the nanoscale: Energetics and length scales of vacancy and interstitial absorption by grain boundaries in alpha-Fe, Phys. Rev. B 85 (2012).

[22] D. Wolf. Correlation Between the Energy and Structure of Grain-Boundaries in BCC Metals 1. Symetrical Boundaries on the (110) and (100) Planes, Phil. Mag. B 59 (1989) 667680.

[23] D. Wolf. Correlation Between the Energy and Structure of Grain-Boundaries in BCC Metals 2. Symetrical Tilt Boundaries, Phil. Mag. A 62 (1990) 447-464.

[24] D. Wolf. Structure and Energy of General Grain-Boundaries in BCC Metals, J. Appl. Phys. 69 (1991) 185-196.

[25] D. Yesilleten, T.A. Arias. Atomic-level physics of grain boundaries in bcc molybdenum, Phys. Rev. B 64 (2001).

[26] A. Morawiec. Tilt and twist grain boundaries, J. Appl. Cryst. 42 (2009) 308-311.

[27] A. Morawiec. Low-Sigma twist and tilt grain boundaries in cubic materials, J. Appl. Cryst. 44 (2011) 1152-1156.

[28] A. Morawiec. On 'interface-plane scheme' and symmetric grain boundaries, Z. Krist. 227 (2012) 199-206.

[29] S.J. Plimpton. LAMMPS: Large-scale Atomic/Molecular Massively Parallel Simulator. Sandia National Laboratories, 2007.

[30] M.I. Mendelev, S. Han, D.J. Srolovitz, G.J. Ackland, D.Y. Sun, M. Asta. Development of new interatomic potentials appropriate for crystalline and liquid iron, Phil.l Mag. 83 (2003) 3977-3994.

[31] M.W. Finnis, J.E. Sinclair. A Simple Empirical N-Body Potential for Transition-Metals, Phil. Mag. A 50 (1984) 45-55.

[32] D.L. Olmsted. unpublished.

[33] T. Frolov, D.L. Olmsted, M. Asta, Y. Mishin. Structural phase transformations in metallic grain boundaries, Nat. Comm. 4 (2013).

[34] J.Q. Broughton, G.H. Gilmer. Interface Melting - Simulations of Surfaces and GrainBoudnaries at High-Temperatures, J. Phys. Chem. 91 (1987) 6347-6359.

[35] J.Q. Broughton, G.H. Gilmer. Harmonic analysis of Lennard-Jones FCC grain boundaries, Modelling Simul. Mater. Sci. Eng. 6 (1998) 393-404.

[36] S.M. Foiles. Evaluation of Harmonic Methods for Calculating the Free-Energy of Defects in Solids, Phys. Rev. B 49 (1994) 14930-14938.

[37] S.M. Foiles. Temperature dependence of grain boundary free energy and elastic constants, Scripta Mater. 62 (2010) 231-234.

[38] P.R. Cantwell, M. Tang, S.J. Dillon, J. Luo, G.S. Rohrer, M.P. Harmer. Grain boundary complexions, Acta Mater.62 (2014) 1-48.

[39] D.L. Olmsted, D. Buta, A. Adland, S.M. Foiles, M. Asta, A. Karma. Dislocation-Pairing Transitions in Hot Grain Boundaries, Phys. Rev. Lett. 106 (2011).

[40] Handbook of Chemistry and Physics. 94th ed., CRC Press, 2013.

[41] C. Kittel. Introduction to Solid State Physics. 5th ed., Wiley, New York, 1976.

[42] S. Ryu, W. Cai. Comparison of thermal properties predicted by interatomic potential models, Modelling Simul. Mater. Sci. Eng. 16 (2008).

[43] J.A. Moriarty. Denisty-Functional Formulation of the Generalized Pseudopotential Theory 2, Phys. Rev. B 26 (1982) 1754-1780. 
[44] J.A. Moriarty. Angular Forces and Melting in BCC Transition-Metals - A Case Study of Molybdenum, Phys. Rev. B 49 (1994) 12431-12445.

[45] S.K. Bhattacharya, S. Tanaka, Y. Shiihara, M. Kohyama. Ab initio study of symmetrical tilt grain boundaries in bcc Fe: structural units, magnetic moments, interfacial bonding, local energy and local stress, J. Phys. - Condensed Matter 25 (2013).

[46] M. Cak, M. Sob, J. Hafner. First-principles study of magnetism at grain boundaries in iron and nickel, Phys. Rev. B 78 (2008).

[47] Y.A. Du, L. Ismer, J. Rogal, T. Hickel, J. Neugebauer, R. Drautz. First-principles study on the interaction of $\mathrm{H}$ interstitials with grain boundaries in alpha- and gamma-Fe, Phy. Rev. B 84 (2011).

[48] N. Gao, C.-C. Fu, A. Samaras, R. Schaeublin, M. Victoria, W. Hoffelner. Multiscale modelling of bi-crystal grain boundaries in bcc iron, J. Nuclear Mater. 385 (2009) 262-267.

[49] T. Ochs, C. Elsasser, M. Mrovec, V. Vitek, J. Belak, J.A. Moriarty. Symmetrical tilt grain boundaries in bcc transition metals: comparison of semiempirical with ab-initio totalenergy calculations, Phil. Mag. A 80 (2000) 2405-2423.

[50] A.M. Tahir, R. Janisch, A. Hartmaier. Ab initio calculation of traction separation laws for a grain boundary in molybdenum with segregated C impurites, Modelling Simul. Mater. Sci. Eng. 21 (2013).

[51] E. Wachowicz, T. Ossowski, A. Kiejna. Cohesive and magnetic properties of grain boundaries in bcc Fe with Cr additions, Phys. Rev. B 81 (2010).

[52] J.-M. Zhang, D.-D. Wang, K.-W. Xu. Calculation of the surface energy of bcc transition metals by using the second nearest-neighbor modified embedded atom method, Appl. Surf. Sci. 252 (2006) 8217-8222.

[53] T.A. Roth. Surface and Grain-Boundary Energies of Iron, Cobalt, and Nickel, Mater. Sci. Eng. 18 (1975) 183-192.

[54] C.G. Dunn, F.W. Daniels, M.J. Bolton. Measurment of Relative Interface Energies in Twin Related Crystals, J. Metals 2 (1950) 368-377.

[55] B.J. Lee, M.I. Baskes, H. Kim, Y.K. Cho. Second nearest-neighbor modified embedded atom method potentials for bcc transition metals, Phys. Rev. B 64 (2001).

[56] C.G. Dunn, F. Lionetti. The Effect of Orientation Differnece on Grain Boundary Energies, Trans. AIMME, 185 (1949) 125-132.

[57] S. Tsurekawa, T. Tanaka, H. Yoshinaga. Grain-Boundary Structure, Energy and Strength in Molybdenum, Mater. Sci. Eng. A 176 (1994) 341-348.

[58] V. Randle, G.S. Rohrer, H.M. Miller, M. Coleman, G.T. Owen. Five-parameter grain boundary distribution of commercially grain boundary engineered nickel and copper, Acta Mater. 56 (2008) 2363-2373.

[59] H. Beladi, G. Rohrer. The Distribution of Grain Boundary Planes in Interstitial Free Steel, Metall and Mat Trans A 44 (2013) 115-124.

[60] D. Wolf. A broken-bond model for grain boundaries in face-centered cubic metals, J. Appl. Phys. 68 (1990) 3221-3236.

[61] P. Wynblatt, Z. Shi. Relation between Grain Boundary Segregation and Grain Boundary Character in FCC alloys, J. Mater. Science 40 (2005) 2765-2773.

[62] P. Wynblatt, M. Takashima. Correlation of Grain Boundary Character with Wetting Behavior, Interface Science 9 (2001) 265-273. 


\section{Figure Captions}

Figure 1. Comparisons of the present results and previously simulated grain boundary energies of $\mathrm{Fe} \mathrm{(a)} \mathrm{and} \mathrm{Mo} \mathrm{(b).} \mathrm{Each} \mathrm{point} \mathrm{corresponds} \mathrm{to} \mathrm{a} \mathrm{particular} \mathrm{grain} \mathrm{boundary} \mathrm{type}$ and the coordinates are the energies computed in the present work and the energies reported previously [19-25]. The solid line has a unit slope. Note that the boundary energy of the $\Sigma 3$ (111) twist boundary, $\Sigma 7$ (111) twist boundary, and $\Sigma 13$ (111) twist boundary calculated by Wolf [22-24] are colored purple, yellow, and brown, respectively. The minimum energy corresponds to the $\Sigma 3$ (112) symmetric tilt boundary.

Figure 2. The calculated boundary energies of Fe (a) and Mo (b). Squares (blue): boundaries with $\{110\}$ planes on both sides. Triangles (red): boundaries with $\{112\}$ planes on both sides. Diamonds, green, yellow, and brown, are boundaries made up from $\{841\}\{221\},\{11,7,1\}\{331\}$, and $\{13,11,5\}\{531\}$ planes, respectively. Open circles are other boundaries.

Figure 3. Calculated grain boundary energies for Fe (a) and Mo (b) plotted versus $\Sigma$, the inverse density of coincident lattice sites. Squares (blue): boundaries with $\{110\}$ planes on both sides. Triangles (red): boundaries with $\{112\}$ planes on both sides. Diamonds, green, yellow, and brown, are boundaries made up from $\{841\}\{221\},\{11,7,1\}\{331\}$, and $\{13,11,5\}\{531\}$ planes, respectively. Open circles are other boundaries.

Figure 4. Calculated grain boundary energies for the $\Sigma 3$ (a \& d), $\Sigma 9$ (b \& e), and $\Sigma 5$ (c \& f) misorientations in Fe $(\mathrm{a}, \mathrm{b}, \mathrm{c})$ and Mo $(\mathrm{d}, \mathrm{e}, \mathrm{f})$, plotted in stereographic projection. The [100] direction is horizontal and to the right, the [010] direction is vertical, and the [001] direction is in the center and perpendicular to the plane of the page. In (a) and (d), the [111] misorientation axis is marked by a triangle and in the others, the misorientation axes are in the plane of the page and marked by the arrows.

Figure 5. The relationship between the calculated boundary energies for Fe and Mo. Each point corresponds to two crystallographically identical grain boundaries in two different materials. The horizontal and vertical axes show the boundary energy in Fe and Mo respectively. Note that the data are colored according to the grain boundary types: (110) symmetrical boundaries (green circles), $\Sigma 3$ boundaries (red squares), the coherent twin boundary (black square), and the other type of boundaries (blue diamonds). Lines show scaling factors predicted by the various materials parameters. 

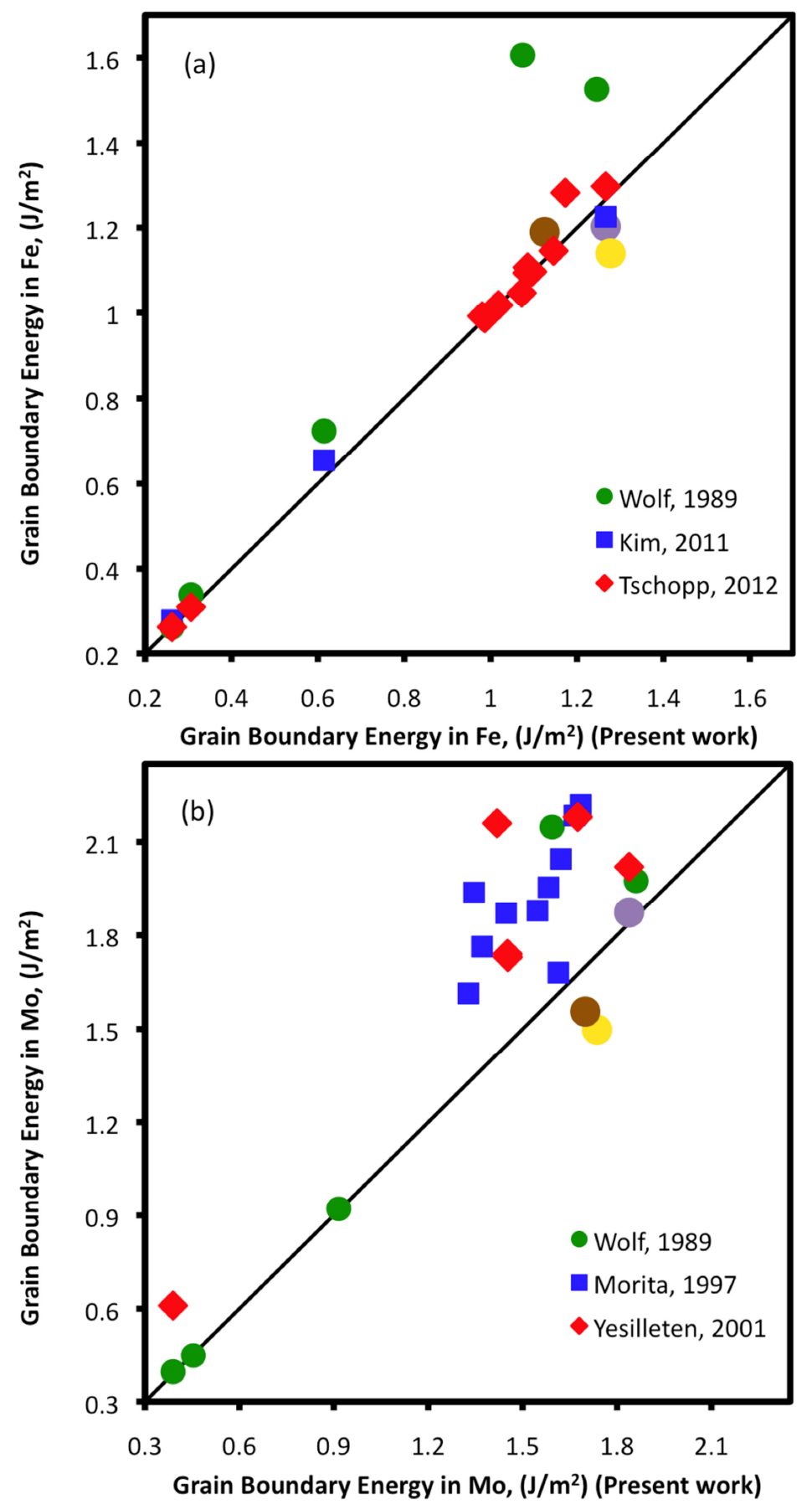

Fig. 1 

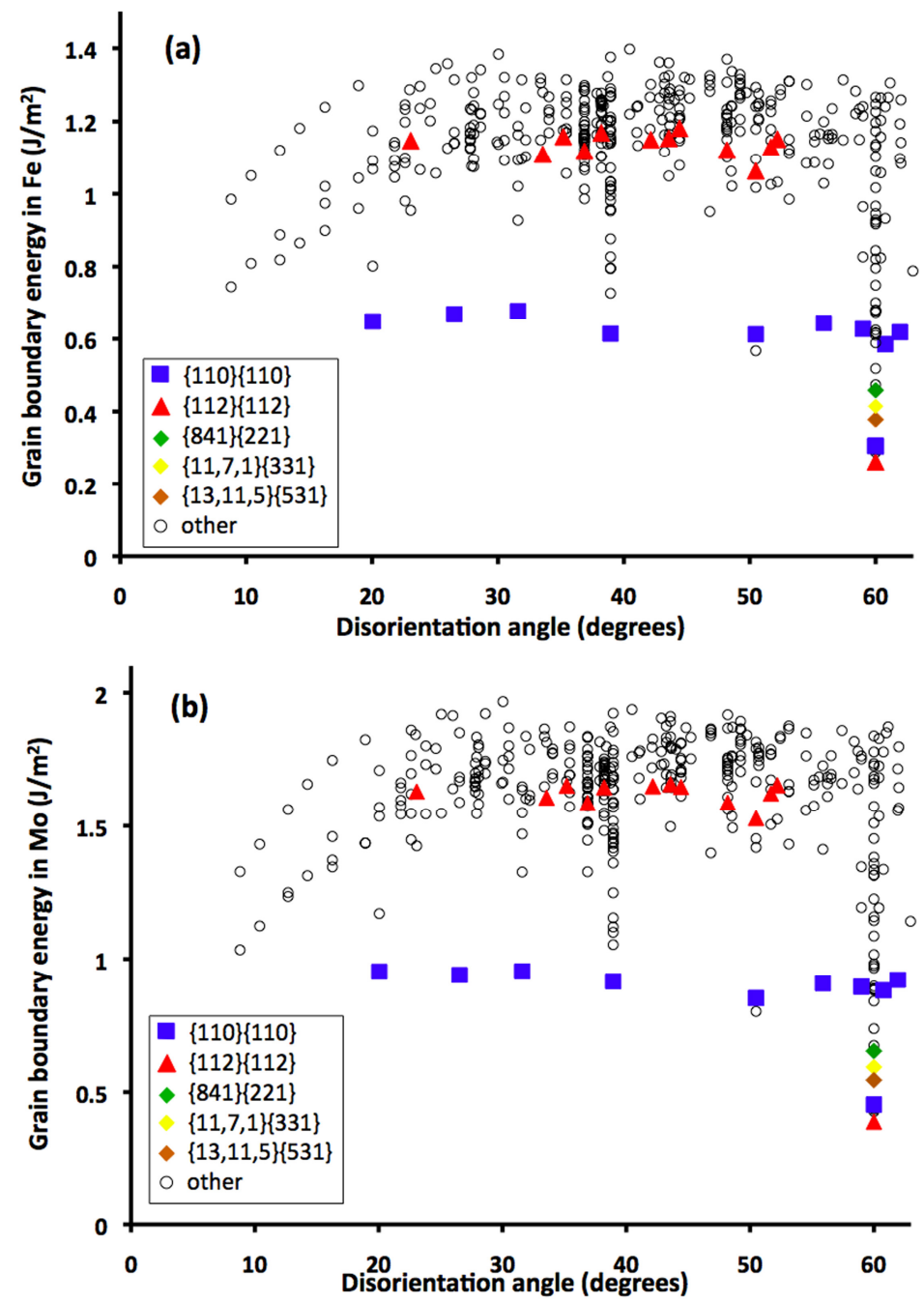

Fig. 2 

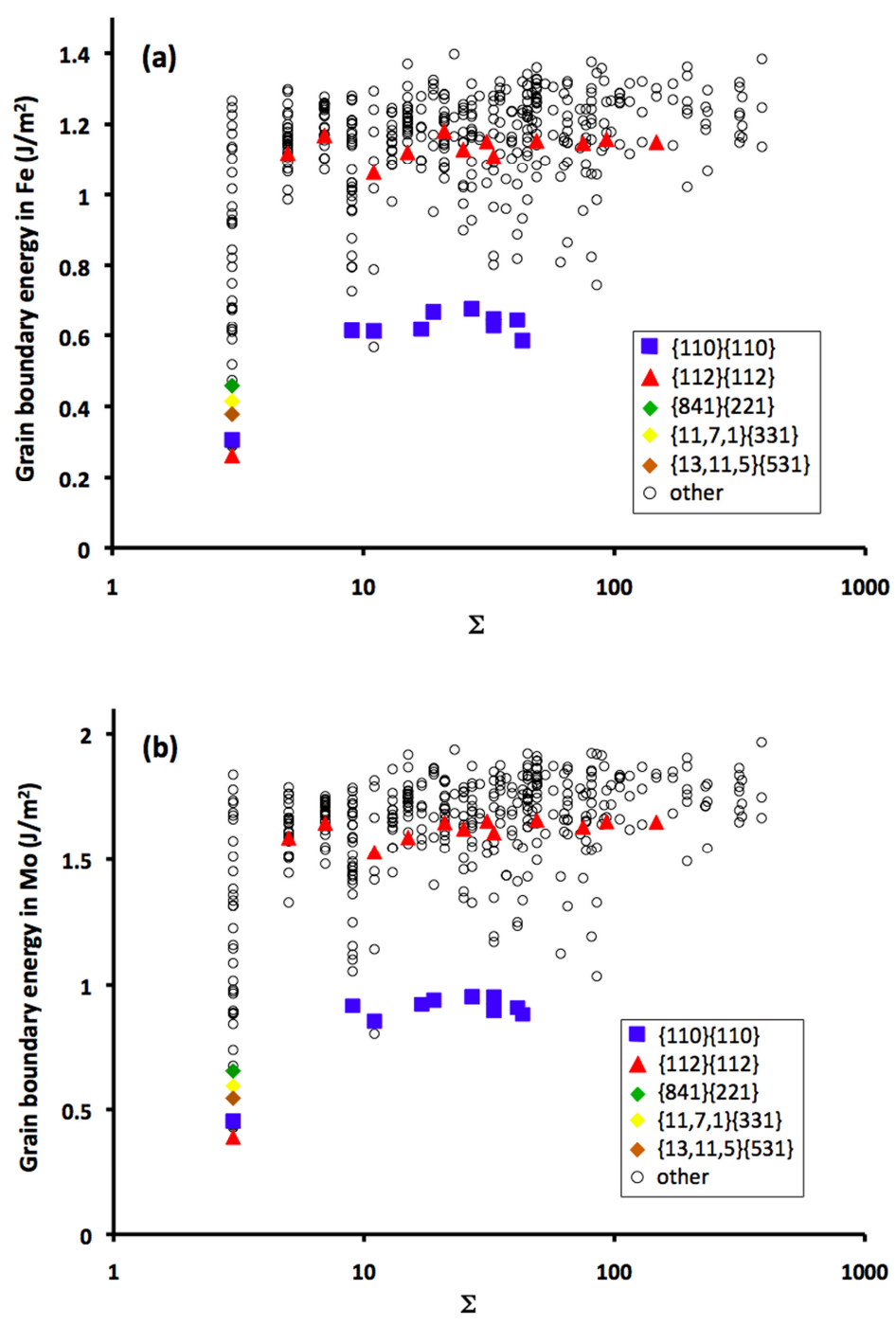

Fig. 3 
$\begin{array}{llllllllllll}0.3 & 0.4 & 0.5 & 0.6 & 0.7 & 0.8 & 0.9 & 1.0 & 1.1 & 1.2 & 1.3\end{array}$

(a) $\Sigma$ 3, Fe, Energy, $\mathrm{J} / \mathrm{m}^{2}$

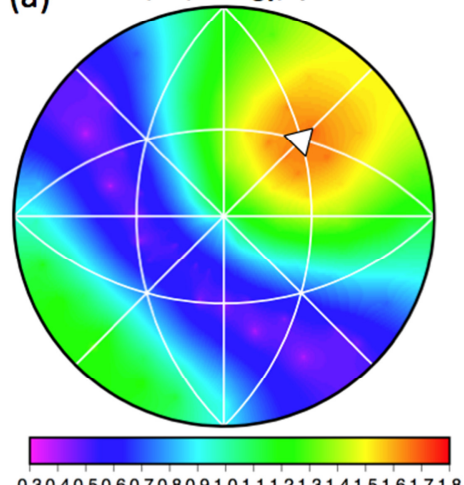

0.30 .40 .50 .60 .70 .80 .91 .01111 .21 .31 .41 .51 .61 .71 .8

(d) $\quad \Sigma 3, \mathrm{Mo}$, Energy, $\mathrm{J} / \mathrm{m}^{2}$

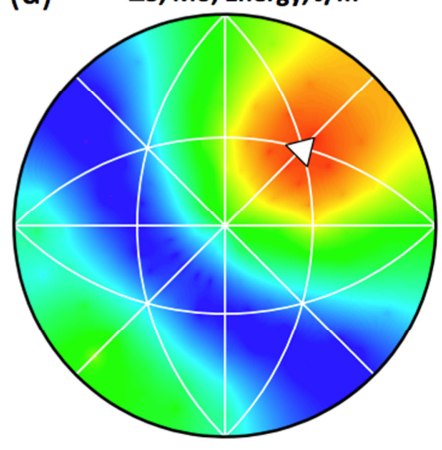

0.750 .800 .850 .900 .951 .001 .051 .101 .151 .201 .251 .30

(b) $\Sigma 9, \mathrm{Fe}$, Energy, $\mathrm{J} / \mathrm{m}^{2}$

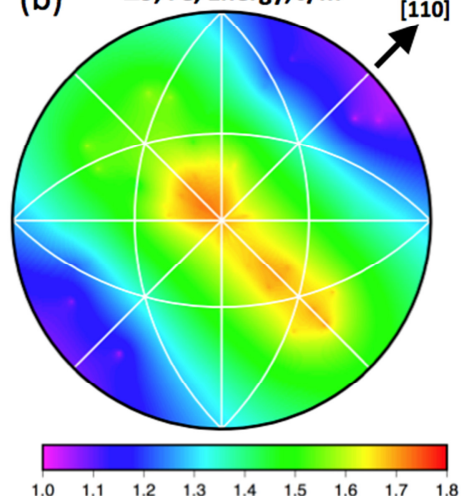

(e) $\Sigma 9$, Mo, Energy, $\mathrm{J} / \mathrm{m}^{2}$

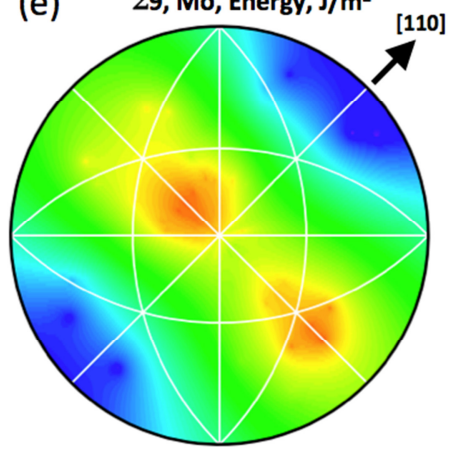

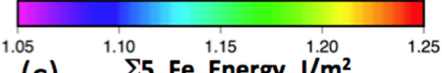

(c) $\quad \stackrel{1.10}{\Sigma 5}, \mathrm{Fe}$, Energy, $\mathrm{J}^{1.20} \mathrm{~m}^{2}$
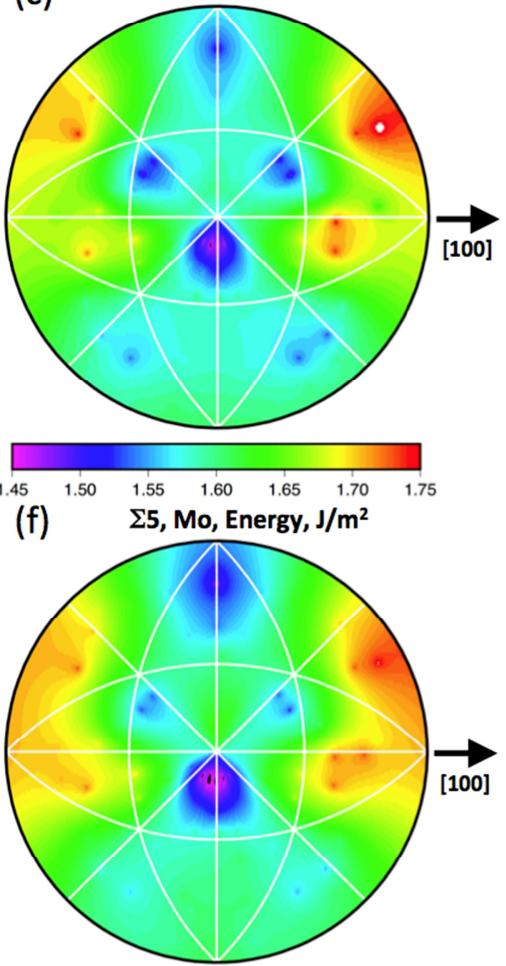

Fig. 4 


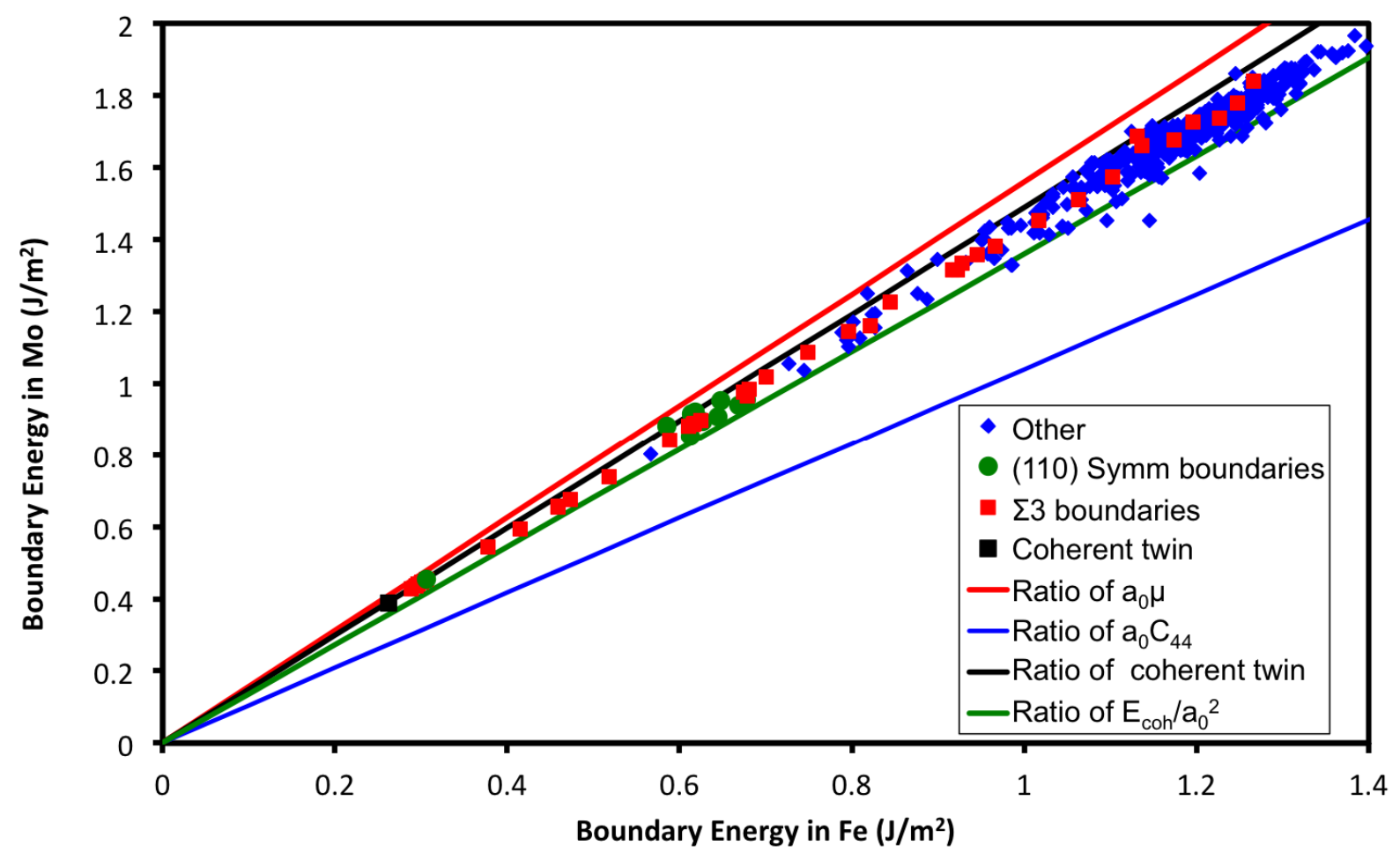

Fig. 5 
Table 1. Selected materials properties for Fe and Mo.

\begin{tabular}{|c|c|c|c|c|}
\hline Materials properties & $\begin{array}{c}\text { Mendelev Fe } \\
\text { EAM2 a }\end{array}$ & $\begin{array}{l}\text { Experimental } \\
\text { values of } \mathrm{Fe}^{\mathrm{b}}\end{array}$ & $\begin{array}{l}\text { Finnis-Sinclair } \\
\text { Mo EAM c }^{c}\end{array}$ & $\begin{array}{l}\text { Experimental } \\
\text { values of } \mathrm{Mo}^{\mathrm{b}}\end{array}$ \\
\hline $\begin{array}{c}\text { Lattice constant } \\
\mathrm{a}_{0}(\AA)\end{array}$ & 2.8553 & 2.8664 & 3.1472 & 3.1470 \\
\hline Melting point $\mathrm{T}_{\mathrm{m}}(\mathrm{K})$ & 1773 & 1811 & $3062.6 \pm 7.6$ & 2896 \\
\hline $\begin{array}{c}\text { Cohesive energy } \\
\mathrm{E}_{\mathrm{coh}}(\mathrm{eV})\end{array}$ & 4.122 & 4.28 & 6.82 & 6.82 \\
\hline $\begin{array}{l}\text { Coherent twin } \\
\text { energy }\left(\mathrm{mJ} / \mathrm{m}^{2}\right)\end{array}$ & 26.2 & -- & 38.9 & - \\
\hline $\begin{array}{l}\text { Bulk modulus } \\
\text { B (GPa) }\end{array}$ & 177.8 & 168.7 & 262.6 & 259.8 \\
\hline $\begin{array}{l}\text { Voigt average shear } \\
\text { modulus } \mu_{\text {voigt }}(\mathrm{GPa})\end{array}$ & 89.28 & 86.8 & 125.98 & 126.7 \\
\hline $\mathrm{C}^{\prime}(\mathrm{GPa})$ & 49.2 & 43 & 151.6 & 153.0 \\
\hline $\mathrm{C}_{11}(\mathrm{GPa})$ & 243.4 & 226 & 464.7 & 463.7 \\
\hline $\mathrm{C}_{12}(\mathrm{GPa})$ & 145.0 & 140 & 161.5 & 157.8 \\
\hline $\mathrm{C}_{44}(\mathrm{GPa})$ & 116.0 & 116 & 108.9 & 109.2 \\
\hline
\end{tabular}

a Simulated materials properties of Fe are from Refs. [30, 37].

b Experimental materials properties of Fe and Mo are from Ref. [38] except for $\mathrm{E}_{\mathrm{coh}}$ from Ref. [39]. c Simulated materials properties of Mo are from Ref. [31] except for $\mathrm{T}_{\mathrm{m}}$ from Ref. [40] 
Table 2. Average energies of grain boundaries at fixed $\Sigma$

\begin{tabular}{cccccc}
\hline$\Sigma$ & $\begin{array}{c}\text { Number } \\
\text { of GBs }\end{array}$ & Ave. Energy, Fe, J/m $\mathrm{m}^{2}$ & std. dev. Fe & Ave. Energy, Mo, J/m $\mathrm{m}^{2}$ & std. dev. Mo \\
\hline 3 & 40 & 1.06 & 0.247 & 1.50 & 0.347 \\
5 & 27 & 1.11 & 0.171 & 1.58 & 0.230 \\
7 & 21 & 0.998 & 0.322 & 1.43 & 0.453 \\
9 & 29 & 1.17 & 0.166 & 1.66 & 0.234 \\
11 & 9 & 1.11 & 0.198 & 1.58 & 0.276 \\
13 & 15 & 1.22 & 0.118 & 1.73 & 0.160 \\
15 & 19 & 1.14 & 0.195 & 1.63 & 0.269 \\
17 & 9 & 1.18 & 0.088 & 1.68 & 0.115 \\
19 & 8 & 1.02 & 0.231 & 1.45 & 0.324 \\
21 & 17 & 1.06 & 0.196 & 1.52 & 0.280 \\
23 & 3 & 1.16 & 0.067 & 1.67 & 0.062 \\
25 & 12 & 1.12 & 0.236 & 1.59 & 0.348 \\
27 & 15 & 1.05 & 0.212 & 1.50 & 0.297 \\
29 & 3 & 1.22 & 0.093 & 1.71 & 0.115 \\
31 & 3 & 0.997 & 0.176 & 1.41 & 0.242 \\
33 & 13 & 1.06 & 0.251 & 1.50 & 0.350 \\
35 & 8 & 1.14 & 0.060 & 1.64 & 0.084 \\
37 & 3 & 0.857 & 0.488 & 1.23 & 0.685 \\
39 & 6 & 1.03 & 0.368 & 1.51 & 0.529 \\
41 & 6 & 1.26 & 0.053 & 1.77 & 0.064 \\
43 & 6 & 1.12 & 0.188 & 1.60 & 0.254 \\
45 & 17 & 1.10 & 0.229 & 1.58 & 0.325 \\
47 & 3 & 0.955 & 0.275 & 1.38 & 0.414 \\
49 & 18 & 1.05 & 0.232 & 1.49 & 0.327 \\
All & 408 & 1.11 & 0.209 & 1.59 & 0.293
\end{tabular}

